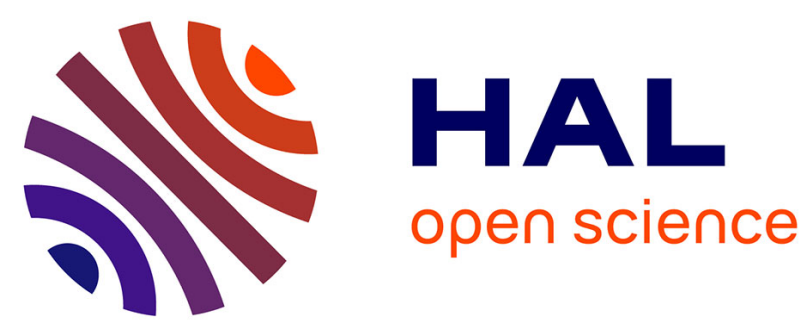

\title{
Simultaneous measurement of gravity acceleration and gravity gradient with an atom interferometer
}

F. Sorrentino, Andrea Bertoldi, Quentin Bodart, L. Cacciapuoti, M. de Angelis, Y.-H. Lien, M. Prevedelli, G. Rosi, G. M. Tino

\section{- To cite this version:}

F. Sorrentino, Andrea Bertoldi, Quentin Bodart, L. Cacciapuoti, M. de Angelis, et al.. Simultaneous measurement of gravity acceleration and gravity gradient with an atom interferometer. Applied Physics Letters, 2012, 101, pp.114106. 10.1063/1.4751112 . hal-00822350

\section{HAL Id: hal-00822350 \\ https://hal-iogs.archives-ouvertes.fr/hal-00822350}

Submitted on 16 Nov 2015

HAL is a multi-disciplinary open access archive for the deposit and dissemination of scientific research documents, whether they are published or not. The documents may come from teaching and research institutions in France or abroad, or from public or private research centers.
L'archive ouverte pluridisciplinaire HAL, est destinée au dépôt et à la diffusion de documents scientifiques de niveau recherche, publiés ou non, émanant des établissements d'enseignement et de recherche français ou étrangers, des laboratoires publics ou privés. 


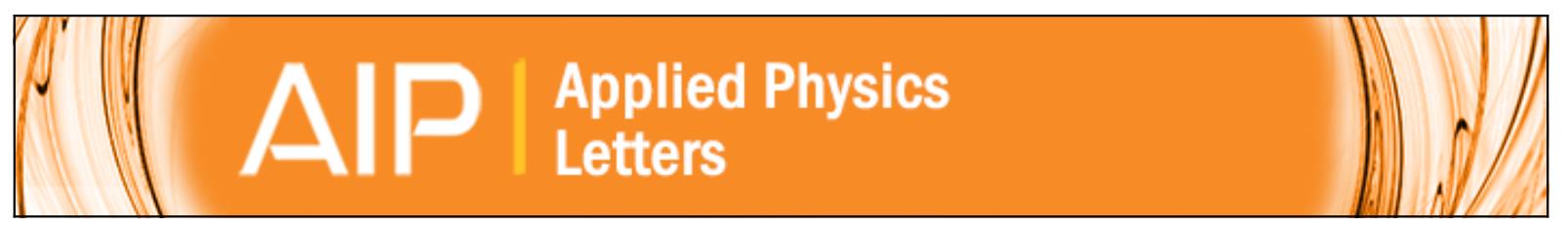

\section{Simultaneous measurement of gravity acceleration and gravity gradient with an atom interferometer \\ F. Sorrentino, A. Bertoldi, Q. Bodart, L. Cacciapuoti, M. de Angelis, Y.-H. Lien, M. Prevedelli, G. Rosi, and G. M. Tino}

Citation: Applied Physics Letters 101, 114106 (2012); doi: 10.1063/1.4751112

View online: http://dx.doi.org/10.1063/1.4751112

View Table of Contents: http://scitation.aip.org/content/aip/journal/apl/101/11?ver=pdfcov

Published by the AIP Publishing

\section{Articles you may be interested in}

Note: Directly measuring the direct digital synthesizer frequency chirp-rate for an atom interferometer

Rev. Sci. Instrum. 86, 096108 (2015); 10.1063/1.4930562

Measuring the Newtonian constant of gravitation with a differential free-fall gradiometer: A feasibility study

Rev. Sci. Instrum. 85, 044501 (2014); 10.1063/1.4869875

High data-rate atom interferometer for measuring acceleration

Appl. Phys. Lett. 100, 011106 (2012); 10.1063/1.3673845

Multiphoton- and simultaneous conjugate Ramsey-Bordé atom interferometers

AIP Conf. Proc. 977, 291 (2008); 10.1063/1.2902792

Development of a simulator of a satellite-to-satellite interferometer for determination of the Earth's gravity field

Rev. Sci. Instrum. 76, 124501 (2005); 10.1063/1.2140280

\section{AIP $\left.\right|_{\text {APL Photonics }}$}

APL Photonics is pleased to announce Benjamin Eggleton as its Editor-in-Chief

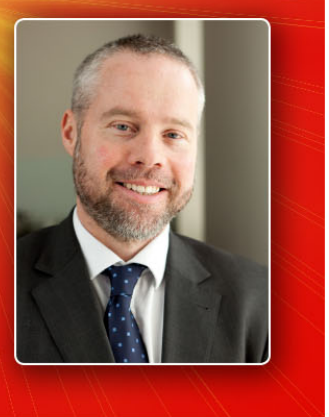




\title{
Simultaneous measurement of gravity acceleration and gravity gradient with an atom interferometer
}

\author{
F. Sorrentino, ${ }^{1}$ A. Bertoldi, ${ }^{2}$ Q. Bodart, ${ }^{1,3}$ L. Cacciapuoti, ${ }^{3}$ M. de Angelis, ${ }^{4}$ Y.-H. Lien, ${ }^{1}$ \\ M. Prevedelli, ${ }^{5}$ G. Rosi, ${ }^{1}$ and G. M. Tino ${ }^{1, a)}$ \\ ${ }^{1}$ Dipartimento di Fisica e Astronomia \& LENS, Università di Firenze, INFN Sezione di Firenze, via Sansone 1, \\ I-50019 Sesto Fiorentino (FI), Italy \\ ${ }^{2}$ Laboratoire Charles Fabry de l'Institut d'Optique, CNRS and Université Paris-Sud Campus Polytechnique, \\ RD 128, F-91127 Palaiseau cedex, France \\ ${ }^{3}$ European Space Agency, Research and Scientific Support Department, Keplerlaan 1, 2201 AZ Noordwijk, \\ The Netherlands \\ ${ }^{4}$ Istituto di Fisica Applicata "Nello Carrara" CNR, via Madonna del Piano 10, I-50019 Sesto Fiorentino (FI), \\ Italy \\ ${ }^{5}$ Dipartimento di Fisica dell' Università di Bologna, Via Irnerio 46, I-40126, Bologna, Italy
}

(Received 28 June 2012; accepted 22 August 2012; published online 12 September 2012)

\begin{abstract}
We demonstrate a method to measure the gravitational acceleration with a dual cloud atom interferometer; the use of simultaneous atom interferometers reduces the effect of seismic noise on the gravity measurement. At the same time, the apparatus is capable of accurate measurements of the vertical gravity gradient. The ability to determine the gravity acceleration and gravity gradient simultaneously and with the same instrument opens interesting perspectives in geophysical applications. (C) 2012 American Institute of Physics. [http://dx.doi.org/10.1063/1.4751112]
\end{abstract}

Atom interferometry has been proven effective for precise measurements of gravity acceleration, ${ }^{1-5}$ Earth's gravity gradient, ${ }^{6-8}$ and rotations. ${ }^{9-12}$

Gravimeters and gravity gradiometers based on atom interferometry are particularly attractive for a number of applications when compared with traditional sensors, because of their excellent long-term stability. ${ }^{13}$ These include metrology, geodesy, geophysics, engineering prospecting, and inertial navigation. ${ }^{7,13-20}$

Absolute gravimeters generally require a high degree of isolation from vertical acceleration noise which, as a consequence of the equivalence principle, cannot be distinguished from gravity itself. This is usually achieved with complex seismic isolation systems, which limit the reliability and compactness of the instrument, two critical issues for field applications. In the case of atom interferometers, where the transfer function of seismic noise to the output of the gravimeter is precisely modeled, the seismic noise can be rejected to some degree by calculating a correction from the reading of a mechanical accelerometer. ${ }^{12}$

On the contrary, atom interferometry based gravity gradiometers are highly immune from vibrations and seismic noise, which are efficiently rejected as common mode signals in a differential measurement when simultaneous atom interferometers (SAIs) are employed.

Gravity gradiometers are used in geophysical applications either to detect sources of relatively small size, or in situations where vibration noise would hamper absolute gravity measurements-i.e., in airborne or satellite gravity mapping. Moreover, the knowledge of gravity gradient is necessary in reducing the measured gravity acceleration to nearby locations.

In this paper, we discuss the use of a gravity gradiometer to also perform a local measurement of the gravitational

a)guglielmo.tino@fi.infn.it. acceleration under severe conditions of vibration noise. This is possible employing one of the two atomic clouds of the gravity gradiometer to sense the acceleration noise. This information can then be used to correct the interferometric phase measured on the second cloud. Such a method is very robust against seismic noise, and allows to use the instrument for simultaneous measurement of gravity acceleration and of the vertical gravity gradient, without need of any seismic isolation or mechanical accelerometer. Our results can have interesting applications both in geophysics, where the knowledge of the gravity gradient is complementary to that of gravity acceleration, and in fundamental physics, where advanced schemes for large-area atom interferometry can be seriously limited by seismic and vibration noise. ${ }^{21}$

The experimental apparatus is described in Refs. 19 and 22. Our gravity gradiometer consists of a dual-cloud matter wave interferometer in which atoms are first cooled and trapped in a magneto-optical trap (MOT) and then launched upwards in a $1 \mathrm{~m}$ long, magnetically shielded vertical tube using the moving molasses technique. Two clouds of ${ }^{87} \mathrm{Rb}$ are trapped and launched in rapid sequence to fly simultaneously on two parabolic trajectories with a constant vertical separation of about $30 \mathrm{~cm}$. In the tube, Raman lasers precisely aligned along the vertical direction excite the atoms on the two-photon Raman transition between the hyperfine levels of the $\mathrm{Rb}$ ground state. Raman transitions are used for velocity selection and initial state preparation of the atomic samples, as well as to realize the interferometric sequence simultaneously for the two atomic clouds, around the apogees of the atomic trajectories. A set of source masses surrounds the atomic fountain, providing a well characterized additional gravity field for the measurement of the gravitational constant $G .^{23}$

In a Raman interferometer, atoms are split, redirected and finally recombined by a $\pi / 2-\pi-\pi / 2$ pulses sequence. ${ }^{1}$ Atom interference fringes are detected by measuring the 
normalized population in one of the two hyperfine levels of the ground state of the Rb atoms. The measured atom interferometer phase depends on the local gravitational acceleration and on the relative phase of Raman lasers, and it is then given by $\phi=\phi_{0}+\phi_{L}+\phi_{N}$, where $\phi_{N}$ is a zero-mean, vibrationinduced phase noise, and $\phi_{L}$ is an extra relative phase of Raman lasers that can be precisely tuned to scan the atom interference fringes; the gravity-induced phase term is given by

$$
\phi_{0}=\left(k_{\text {eff }} g-\alpha\right) T^{2}
$$

where $k_{\text {eff }}$ is the effective wave vector of the Raman transition, $g$ is the gravity acceleration, $\alpha$ is the frequency chirp rate applied to the Raman lasers frequency difference to compensate for the varying Doppler shift induced by gravity, and $T$ is the time interval between Raman pulses. For inertial sensing, the atoms must interact with two counter-propagating Raman laser beams. Vibrations of the optics in the uncommon beam paths cause relative phase fluctuations of Raman laser fields, which directly map into phase noise of the interferometer as represented by $\phi_{N}$. Figure 1 shows a typical interferometric fringe for $T=160 \mathrm{~ms}$ when scanning $\phi_{L}$ without seismic isolation. Each point represents a couple of values $\left(x^{i}, \phi_{L}^{i}\right)$ where $x^{i}$ is the normalized population of the $F=1$ level, and $\phi_{L}^{i}$ is the scanned relative phase of Raman lasers.

A direct least-squares fit of measured points to the function

$$
x=A \sin \left(\phi_{L}+\phi_{0}\right)+B
$$

for retrieving the phase $\phi_{0}$ would clearly yield unreliable results in such conditions of seismic noise. In the presence of large phase noise, but small amplitude noise, a prior determination of the fringe amplitude and bias from the probability density function (PDF) of data allows to disentangle phase and amplitude noise, and provides better results for the determination of $\phi_{0} \cdot{ }^{24}$ Assuming the interferometer signal to be given by $x=A \sin y+B+\mathcal{N}\left(0, \sigma^{2}\right)$, where the phase $y$ is a random variable with uniform distribution, and $\mathcal{N}\left(0, \sigma^{2}\right)$ is a Gaussian zero-mean additive amplitude noise with variance $\sigma^{2}$, the model PDF reads

$$
P(x)=\int_{B-A / 2}^{B+A / 2} \frac{1}{\sqrt{A^{2}-(B-y)^{2}}} \frac{A e^{-\frac{(y-x)^{2}}{2 \sigma^{2}}}}{\sqrt{2 \pi} \sigma} d y .
$$

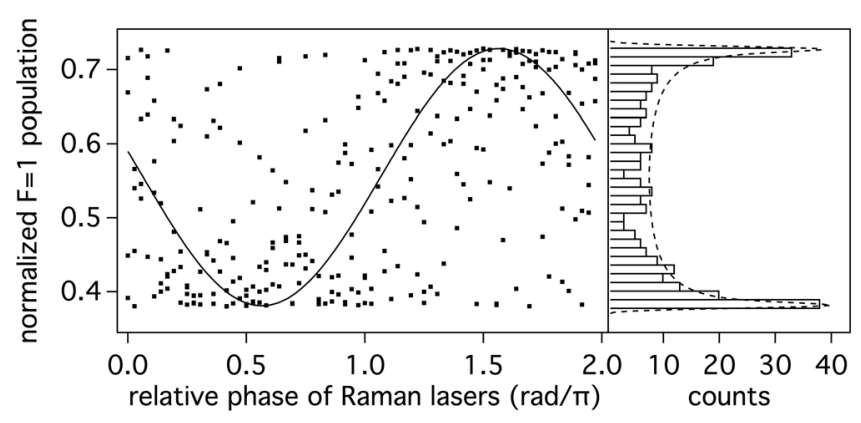

FIG. 1. Left: fringe pattern obtained in a single-cloud interferometer, with a time $T=160 \mathrm{~ms}$ between Raman pulses; each point represents a single measurement; the continuous line is a least-square fit to the data using Eq. (2) after determining the $A$ and $B$ parameters from Eq. (3). Right: histogram of measured data; dashed line is a least-squares fit to the data using Eq. (3).
Once $\mathrm{A}$ and $\mathrm{B}$ have been determined from least-squares fit of the $x_{i}$ distribution to $P(x)$, the interferometer phase $\phi_{0}$ can be retrieved by fitting the measurements $\left\{x^{i}, \phi_{L}^{i}\right\}$ to Eq. (2), with only $\phi_{0}$ as a free parameter. Alternatively, it is possible to invert equation (2) to retrieve the phase $\phi^{i}$ of each individual measured point as

$$
\phi^{i}=\arcsin \frac{x^{i}-B}{A}-\phi_{L}^{i},
$$

where $A$ and $B$ are determined through Eq. (3); then $\phi_{0}$ is obtained from the distribution of the calculated $\phi^{i}$ values, assuming that $\phi_{N}$ is normally distributed. Such algorithm is not able to solve the $\pi$ phase ambiguity, as all points whose phase differs by integer multiples of $\pi$ are folded back in the $\left[-\frac{\pi}{2},+\frac{\pi}{2}\right]$ interval. If the variance of $\phi_{N}$ is much larger than $\pi$, the $\phi_{i}$ distribution is flat and gives no information about the average value $\phi_{0}$.

In the SAIs configuration, two vertically separated lightpulse atom interferometers share the same pair of Raman lasers $^{6-8}$ and measure the local acceleration with respect to the common reference frame identified by the wave fronts of the Raman lasers. The phases of upper and lower interferometer, respectively, are then given by $\phi_{x}=\phi$ and $\phi_{y}=\phi+\Delta \phi$ where $\Delta \phi$ is the phase difference due to vertical gravity gradient. Therefore, even when the phase noise induced by seismic activities and vibrations washes out the atom interference fringes, the signals simultaneously detected on the upper and lower accelerometers remain correlated and preserve a fixed phase relation. As a consequence, when the trace of the upper accelerometer is plotted as a function of the lower one, experimental points $\left\{x^{i}, y^{i}\right\}$ distribute along a Lissajous ellipse. The ellipse best fitting of the experimental data ${ }^{25}$ can be expressed with the parametric equations

$$
\left\{\begin{array}{l}
x=A \sin \phi+B \\
y=C \sin (\phi+\Delta \phi)+D .
\end{array}\right.
$$

Here, $A, B, C$, and $D$ represent the amplitude and offset of the two atom interferometer fringes, while $\phi$ is the phase due to common acceleration and seismic noise. Ellipse fitting yields the phase offset $\Delta \phi$ which is a measure of the differential gravity acceleration.

However, the same algorithm can be used to determine the gravity acceleration, i.e., the phase $\phi_{0}$. In fact, least squares ellipse fitting also provides the best estimate for the actual phase $\phi^{i}$ of each individual measured point. The distribution of $\phi^{i}$ values is folded in the $[-\pi ;+\pi]$ interval. Figure 2 shows the distribution of $\phi^{i}$ data obtained from a single fringe and from ellipse fitting, corresponding to the fringes in Figure 3. As long as the RMS phase noise is not much higher than the amplitude of the phase folding interval, the phase $\phi_{0}$ and its standard error can be determined by fitting the distribution of $\phi^{i}$ values with a folded Gaussian function.

In the presence of large phase noise, the use of SAIs offers two main advantages. First, the phase distribution is determined over a range which is twice that of a single interferometer. In addition, when using a single interferometer, the retrieval fidelity of individual phases $\phi^{i}$ is quite poor at the edges of the measurement range, where the fringe slope is minimal and the conversion of amplitude noise into phase 


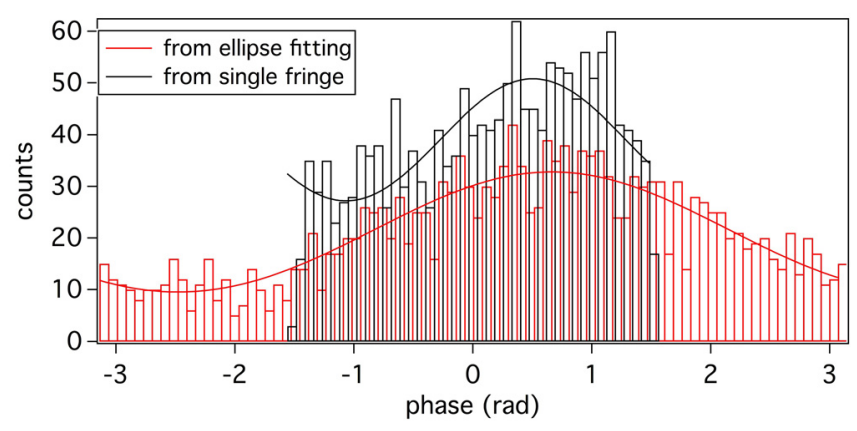

FIG. 2. Histograms of $1800 \phi_{i}$ values with $T=160 \mathrm{~ms}$, calculated according to Eqs. (4) (in black) and (5) (in red); the solid lines represent least-squares fits with folded Gaussians.

noise is maximal. This is not the case with ellipse fitting, where the phase at the edges of the lower interferometer fringe is mainly determined by the signal of the upper interferometer, and vice versa, if the phase difference $\Delta \phi$ is sufficiently large (see below). The last point is illustrated in Figure 3 where we compare the reconstructed fringes obtained by the two methods. To test the single-fringe phase retrieval, the lower interferometer signal is plotted versus the phase calculated on the upper interferometer from Eq. (4). In the case of perfect fidelity, the phases of the two interferometers should have a constant difference $\Delta \phi$. However, when setting $\phi_{y}^{i}=\phi_{x}^{i}$, the fringe of lower interferometer is sensibly noisier than when using ellipse fitting. For unambiguous retrieval of $\phi^{i}$ along the whole range with ellipse fitting, the minimal radius of curvature of the ellipse must be larger than the RMS amplitude noise (i.e., the $\sigma$ parameter in Eq. (3)). This means that the phase difference $\Delta \phi$ between
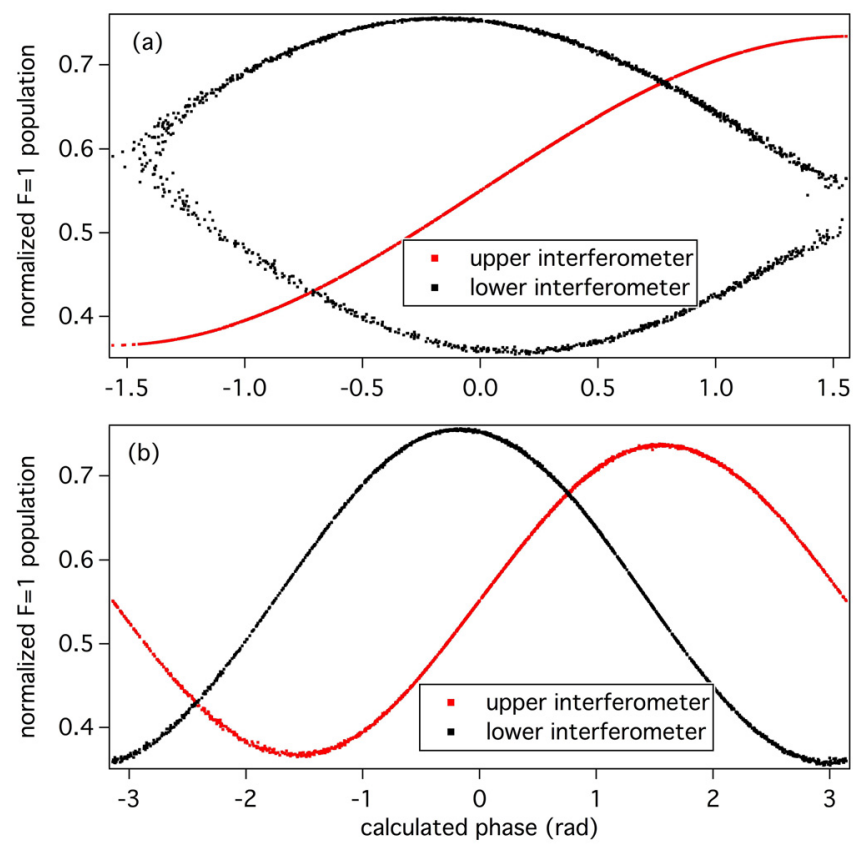

FIG. 3. Reconstructed fringes for $T=160 \mathrm{~ms}$ : the measurements $x^{i}$ and $y^{i}$ from the two simultaneous interferometers are plotted versus the calculated phase $\phi^{i}$; each fringe contains 1800 experimental points, and each point represents a single measurement; in (a), the phase $\phi^{i}$ is computed from the upper interferometer by applying Eq. (4) after determining $A$ and $B$ with Eq. (3); in (b), $\phi^{i}$ is computed with Eq. (5). the two interferometers must be larger than the signal-tonoise ratio $\sqrt{A C} / \sigma$. With our typical values of contrast and amplitude noise, this requirement reads $\Delta \phi>15 \mathrm{mrad}$. The phase difference due to the Earth gravity gradient with $T \simeq$ $150 \mathrm{~ms}$ and cloud separation $d \simeq 30 \mathrm{~cm}$ is $\Delta \phi \simeq 386 \mathrm{mrad}$.

In order to show the ability of this technique to measure the gravity acceleration, we recorded the dual interferometer output continuously for about two days. We calculated $\phi_{0}$ from histograms, then $g$ and its standard error through Eq. (1), every 1800 experimental points, i.e., about one hour. The resulting $g$ temporal plot is shown in Fig. 4, where it is compared to the tidal prediction. In the presence of large, seismically induced phase noise, the dual-cloud algorithm is still able to track the tidal gravity changes, while the performance is sensibly worse with a single cloud. When comparing the two methods, the short-term sensitivity is a factor two better with ellipse fitting; however, when fitting the $g$ data to the tidal model using a baseline as the only free parameter, the RMS of residuals amount to $\sim 13 \mu$ gal and $\sim 36 \mu$ gal respectively, i.e., it is three times smaller with SAIs than with a single fringe. Using three ellipses with slightly different value of the interferometer time $T$, in order to determine the fringe order, we derive an absolute value for the gravity acceleration $g=9.804972 \pm 0.000079 \mathrm{~m} / \mathrm{s}^{2}$, in good agreement with the value of 9.80492048 $\pm 0.00000003 \mathrm{~m} / \mathrm{s}^{2}$ measured with a commercial FG5 gravimeter in the same location. ${ }^{26}$

While reliable Gaussian fitting requires histograms with a large number of points, the temporal resolution can be improved by using a Bayesian estimator. Let us assume that the actual phase is normally distributed around the unknown value $\phi_{0}$ with variance $\sigma$, and let us neglect the probability for $\phi$ to fall outside the range $[-3 \pi ;+3 \pi]$, while experimental points $\phi_{i}$ are folded in the $[-\pi ;+\pi]$ interval; to determine $\phi_{0}$ and its standard error we employ the Bayesian estimator

$$
\hat{\phi}_{0}=\int_{-\pi}^{+\pi} \frac{1}{2 \pi} \phi_{0} p\left(\left\{\phi_{i}\right\} \mid \phi_{0}\right) d \phi_{0},
$$

where the conditional probability density is given by

$$
p\left(\left\{\phi_{i}\right\} \mid \phi_{0}\right)=N \sum_{n=-1}^{1} e^{-\frac{\sum_{i}\left(\phi_{i}-\phi_{0}-2 n \pi\right)^{2}}{2 \sigma^{2}}}
$$

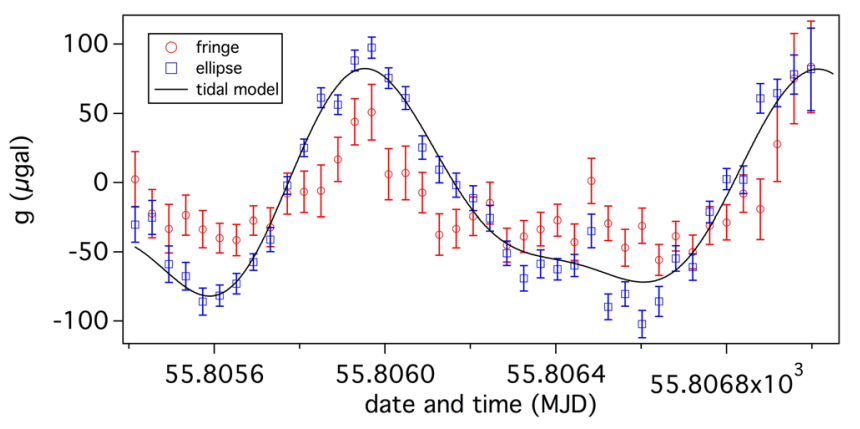

FIG. 4. Variation of Earth's gravity as a function of time. Each dot is obtained by averaging the measured data over a $1 \mathrm{~h}$ interval. Error bars represent the statistical error. The solid line is a fit to predicted Earth's tides with a baseline as the only free parameter. 
and $N$ is a normalization constant. With this technique, we can obtain a single $g$ value after recording $\sim 70$ experimental points, i.e., after about two minutes.

The Allan deviation of the difference between measured and predicted $g$ values rolls off as $7 \times 10^{-6} \mathrm{~ms}^{-2} / \sqrt{\tau}$ up to $90 \mathrm{~min}$, reaching a level of about $13 \mu \mathrm{gal}$. The sensitivity is somehow limited by the repetition rate of the experimental sequence, which cannot be larger than $0.5 \mathrm{~Hz}$ due to geometrical constraints in our apparatus which is specifically designed for the $G$ measurement.

The sensitivity of differential gravity measurement, as obtained from the Allan deviation of $\Delta \phi$ data, is about $9 \times 10^{-8} \mathrm{~ms}^{-2} / \sqrt{\mathrm{Hz}}$. From the measurement of $\Delta \phi$ and of the clouds separation $d$, we derive the absolute value of the gravity gradient as $\gamma=\Delta \phi /\left(d k_{e f f} g T^{2}\right)$. The contribution of residual magnetic field gradient has been evaluated to $(5.0 \pm 0.5) \times 10^{-8} \mathrm{~s}^{-2}$, by measuring the ellipse phase angle versus the applied bias field in the region of the atom interferometry sequence, and extrapolating to zero field. We estimate the effect of the Coriolis acceleration by measuring the average horizontal velocities of the two atomic clouds along the eastwest direction with a precision of $\sim 0.1 \mathrm{~mm} / \mathrm{s} .{ }^{27}$ Finally, after correcting for the gravity gradient produced by the closest masses, ${ }^{26}$ we obtain $\gamma=(3.13 \pm 0.03) \times 10^{-6} \mathrm{~s}^{-2}$, in fair agreement with the standard free-air value of $3.09 \times 10^{-6} \mathrm{~s}^{-2}$. The uncertainty is dominated by the Coriolis shift, which, however, can be efficiently suppressed with the use of a tip-tilt mirror. ${ }^{28}$ The remaining contributions amount to $\sim 7 \times 10^{-9} \mathrm{~s}^{-2}$.

In conclusion, we demonstrated the combined measurement of gravity gradient and gravity acceleration using a pair of simultaneous atom interferometers. The instrument can operate without need for seismic attenuation with a RMS phase noise of $\sim 1 \times \pi$. The use of a mechanical accelerometer in combination with a single-cloud atom interferometer ${ }^{12}$ would be compatible with comparatively larger phase noise. Nevertheless, under typical seismic noise, reliable operation of our method is possible with an interferometer time $T \sim 150 \mathrm{~ms}$ using a clouds separation of $\sim 30 \mathrm{~cm}$. Such a configuration is especially suited for the realization of compact devices for field use. Moreover, our method might be extended to the case of SAIs with different scale factors, i.e., with different atomic species, ${ }^{29,30}$ where the range for phase retrieval could be largely expanded.

This work was supported by INFN (MAGIA experiment), EU (iSense STREP Project Contract No. 250072), and ESA (SAI project Contract No. 20578/07/NL/VJ). F.S. and M.d.A. acknowledge financial support from ESF through the Euroquasar program. The authors thank M. Falorsi for technical help.
${ }^{1}$ M. Kasevich and S. Chu, Appl. Phys. B 54, 321 (1992).

${ }^{2}$ A. Peters, K. Y. Chung, and S. Chu, Nature 400, 849 (1999).

${ }^{3}$ H. Müller, S.-W. Chiow, S. Herrmann, S. Chu, and K.-Y. Chung, Phys. Rev. Lett. 100, 031101 (2008).

${ }^{4}$ S. Merlet, Q. Bodart, N. Malossi, A. Landragin, F. P. D. Santos, O. Gitlein, and L. Timmen, Metrologia 47, L9 (2010).

${ }^{5}$ N. Poli, F. Wang, M. G. Tarallo, A. Alberti, M. Prevedelli, and G. M. Tino, Phys. Rev. Lett. 106, 038501 (2011).

${ }^{6}$ M. J. Snadden, J. M. McGuirk, P. Bouyer, K. G. Haritos, and M. A. Kasevich, Phys. Rev. Lett. 81, 971 (1998).

${ }^{7}$ J. M. McGuirk, G. T. Foster, J. B. Fixler, M. J. Snadden, and M. A. Kasevich, Phys. Rev. A 65, 033608 (2002).

${ }^{8}$ A. Bertoldi, G. Lamporesi, L. Cacciapuoti, M. D. Angelis, M. Fattori, T. Petelski, A. Peters, M. Prevedelli, J. Stuhler, and G. M. Tino, Eur. Phys. J. D 40, 271 (2006).

${ }^{9}$ T. L. Gustavson, P. Bouyer, and M. Kasevich, Phys. Rev. Lett. 78, 2046 (1997).

${ }^{10}$ T. L. Gustavson, A. Landragin, and M. Kasevich, Class. Quantum Grav. 17, 2385 (2000).

${ }^{11}$ B. Canuel, F. Leduc, D. Holleville, A. Gauguet, J. Fils, A. Virdis, A. Clairon, N. Dimarcq, C. J. Bordé, A. Landragin, and P. Bouyer, Phys. Rev. Lett. 97, 010402 (2006).

${ }^{12}$ A. Gauguet, B. Canuel, T. Lévèque, W. Chaibi, and A. Landragin, Phys. Rev. A 80, 063604 (2009).

${ }^{13}$ M. de Angelis, A. Bertoldi, L. Cacciapuoti, A. Giorgini, G. Lamporesi, M. Prevedelli, G. Saccorotti, F. Sorrentino, and G. M. Tino, Meas. Sci. Technol. 20, 022001 (2009).

${ }^{14}$ A. Peters, K. Y. Chung, and S. Chu, Metrologia 38, 25 (2001).

${ }^{15}$ A. Bresson, Y. Bidel, P. Bouyer, B. Leone, E. Murphy, and P. Silvestrin, Appl. Phys. B 84, 545 (2006).

${ }^{16} \mathrm{G}$. Geneves, IEEE Trans. Instrum. Meas. 54, 850 (2005).

${ }^{17}$ N. Yu, J. M. Kohel, J. R. Krollog, and L. Maleki, Appl. Phys. B 84, 647 (2006).

${ }^{18}$ M. Fattori, G. Lamporesi, T. Petelski, J. Stuhler, and G. M. Tino, Phys. Lett. A 318, 184 (2003).

${ }^{19}$ G. Lamporesi, A. Bertoldi, L. Cacciapuoti, M. Prevedelli, and G. M. Tino, Phys. Rev. Lett. 100, 050801 (2008).

${ }^{20}$ J. B. Fixler, G. T. Foster, J. M. McGuirk, and M. Kasevich, Science 315, 74 (2007).

${ }^{21}$ S.-W. Chiow, T. Kovachy, H.-C. Chien, and M. A. Kasevich, Phys. Rev. Lett. 107, 130403 (2011).

${ }^{22}$ F. Sorrentino, Y.-H. Lien, G. Rosi, G. M. Tino, L. Cacciapuoti, and M. Prevedelli, New J. Phys. 12, 095009 (2010).

${ }^{23}$ G. Lamporesi, A. Bertoldi, A. Cecchetti, B. Duhlach, M. Fattori, A. Malengo, S. Pettorruso, M. Prevedelli, and G. M. Tino, Rev. Sci. Instrum. 78, 075109 (2007).

${ }^{24}$ R. Geiger, V. Ménoret, G. Stern, N. Zahzam, P. Cheinet, B. Battelier, A. Villing, F. Moron, M. Lours, Y. Bidel, A. Bresson, A. Landragin, and P. Bouyer, Nat. Commun. 2, 474 (2011).

${ }^{25}$ G. T. Foster, J. B. Fixler, J. M. McGuirk, and M. A. Kasevich, Opt. Lett. 27, 951 (2002).

${ }^{26}$ M. de Angelis, F. Greco, A. Pistorio, N. Poli, M. Prevedelli, G. Saccorotti, F. Sorrentino, and G. M. Tino, Eur. Phys. J. Plus 127, 27 (2012).

${ }^{27}$ G. Rosi, Q. Bodart, L. Cacciapuoti, Y.-H. Lien, M. Prevedelli, L. Salvi, F. Sorrentino, and G. M. Tino, "Effect of atomic motion in atom interferometry gravity gradient measurements" (unpublished).

${ }^{28}$ S.-Y. Lan, P.-C. Kuan, B. Estey, P. Haslinger, and H. Müller, Phys. Rev. Lett. 108, 090402 (2012).

${ }^{29}$ G. Varoquaux, R. A. Nyman, R. Geiger, P. Cheinet, and A. Landragin, New J. Phys. 11, 113010 (2009).

${ }^{30}$ W. Ertmer, C. Schubert, T. Wendrich, M. Gilowski, M. Zaiser, T. V. Zoest, E. Rasel, Ch. Bordé, A. Clairon, A. Landragin et al., Exp. Astron. 23, 611 (2009). 